received the name of "oculists," and when Queen Anne came to the throne a great fillip was given to these oculists, as she herself suffered from defective sight, and she also revived the custom of "touching" for King's evil, and took fees in the form of "touch-pieces" from patients. Queen Anne appointed two charlatans as oculists to the Court. One was William Read, a tailor, who subsequently received a knighthood for curing a number of seamen and soldiers gratis, and the other, Roger Grant, either a shoemaker or a tinker. The latter was notorious for " putting out eyes with great success." In this irregular way the now much coveted title of "Surgeon-oculist to the King" arose. About this time the king of all quack oculists, Chevalier Taylor, flourished; he was a qualified surgeon, an account of whose life is given by Coats in the Royal London Ophthalmic Hospital Reports for 1915. He was appointed oculist to the Pope and to the Kings of England, Denmark, Poland, Norway and Sweden, and he used to go about in a carriage painted all over with eyes with the motto on it of "Qui visum dat, vitam dat." He operated on Handel for cataract, " but on drawing the curtain we found the bottom defective."

Coming to later day quacks in this country, they seem to be divided into two classes, foreigners who come from a Germanspeaking country and set up for a time in one of our big hotels, their coming having been duly advertised beforehand, curing every disease of the eye by lotion, drops or glasses. The other, perhaps less reprehensible, is represented by the optician who lays himself out for a course of treatment by strong glasses or other means and charges exorbitant fees for the same. There is still a further class whose activities are usually shortlived, such as the case of the Indian oculist who used to remove pieces of tissue from the conjunctiva, and more recently the beauty specialist who excises redundant skin about the eyelids in old people. Mr. Cyril Walker ends his most interesting lecture by urging the general practitioner actively to assist in the suppression of quackery in ophthalmology by keeping in touch with the nearest eye clinic and sending all cases for consultation, thus not only maintaining his interest in ophthalmology but learning better its possibilities and limitations, enabling him to decide when to persuade his patients to seek special advice rather than allowing them to drift into the hands of the optician or quack. By doing this he will more than ever deserve to be regarded as their guide, philosopher and friend.

\title{
Amaurosis after Loss of Blood
}

Little attention has been paid to this curious phenomenon in the standard text books. The Ophthalmological Society of Paris had an interesting discussion on the subject at a recent meeting 
(an account of this is to be found in the Arch. d'Ophtal. for January, 1922). Dr. A. Terson read the opening paper summing up the various points of interest and the work of previous observers. In the first place it is a rare occurrence in spite of the frequency of the profuse haemorrhages that might give rise to it. In the second place it hardly ever follows immediately on the haemorrhage, but makes its appearance from three to eight days later, and never at a greater interval than three weeks. It is of more frequent occurrence in patients over forty, in women, and in people in a state of bad general health. The usual ophthalmoscopic picture is one of ischaemia of the optic nerve with contracted retinal arteries, and occasionally some oedema of the optic disc and retina. The usual termination is partial or total atrophy of the optic nerve. The affection of sight is frequently accompanied by evidence of participation of other nerves and centres (deafness, oculo-motor paralyses, intense headache, coma, polyneuritis). Terson pointed out that blindness also occurred after haemorrhage in animals.

Retinal ischaemia alone might account for the cases, few in number, that follow directly on the loss of blood, but does not explain the majority of cases that develop at a later period. For those he suggests a process analogous to that seen in anaphylaxis. The fact that the affection is most frequent in patients in a bad state of health lends some support to the theory of a neurotoxaemia, as suggested in a paper by Terrien noticed elsewhere in this journal. Post-mortem examination of cases has shown no signs of inflammation, nor have any embolisms or thromboses been observed. The conditions found have been those of an ascending atrophy of the optic nerve of peripheral origin, accompanied by degenerative retinal lesions. Holden claims to have been able to produce the same results by repeated bleeding in animals.

As regards treatment, in addition to the usual general treatment for haemorrhage, Terson advocates repeated paracenteses of the eye and the use of dionin.

In the subsequent discussion several surgeons gave notes of cases. M. Rochon-Duvigneaud made the interesting observation that veterinary surgeons habitually bled horses suffering from congestive colic to the extent of five or six litres. Blindness, which lasted four or five days sometimes followed this operation. In contrast to this at the Pasteur Institute horses were repeatedly bled to large amounts in the manufacture of serum without any untoward results. Although these horses are old they are in good health, and Rochon-Duvigneaud suggests that the general health of the animal is probably the determining factor. 